\title{
ON MINIMUM DEGREE IMPLYING THAT A GRAPH IS $H$-LINKED*
}

\author{
RONALD J. GOULD ${ }^{\dagger}$, ALEXANDR KOSTOCHKA ${ }^{\ddagger}$ AND GEXIN YU ${ }^{\S}$
}

\begin{abstract}
Given a fixed multigraph $H$, possibly containing loops, with $V(H)=\left\{h_{1}, \ldots, h_{m}\right\}$, we say that a graph $G$ is $H$-linked if for every choice of $m$ vertices $v_{1}, \ldots, v_{m}$ in $G$, there exists a subdivision of $H$ in $G$ such that $v_{i}$ is the branch vertex representing $h_{i}$ (for all $i$ ). This generalizes the concept of $k$-linked graphs (as well as a number of other well-known path or cycle properties). In this paper we determine a sharp lower bound on $\delta(G)$ (which depends upon $H$ ) such that each graph $G$ on at least $10(|V(H)|+|E(H)|)$ vertices satisfying this bound is $H$-linked.
\end{abstract}

Key words. minimum degree, connectivity, $k$-linked, $H$-linked

AMS subject classifications. 05C40, 05C38

DOI. $10.1137 / 050624662$

1. Introduction. For terms not defined here, see [9]. A graph is $k$-linked if for every sequence of $2 k$ vertices, $v_{1}, \ldots, v_{k}, w_{1}, \ldots, w_{k}$, there are internally disjoint paths $P_{1}, \ldots, P_{k}$ such that $P_{i}$ joins $v_{i}$ and $w_{i}$. The literature contains numerous results and important open problems dealing with $k$-linked graphs. In this paper we are concerned with the following generalization of $k$-linked graphs.

Let $H$ be a multigraph. An $H$-subdivision in a graph $G$ is a pair of mappings $f: V(H) \rightarrow V(G)$ and $g: E(H)$ into the set of paths in $G$ such that:

(a) $f(u) \neq f(v)$ for all distinct $u, v \in V(H)$;

(b) for every $u v \in E(H), g(u v)$ is an $f(u), f(v)$-path in $G$, and distinct edges map to internally disjoint paths in $G$.

A graph $G$ is $H$-linked if every injective mapping $f: V(H) \rightarrow V(G)$ can be extended to an $H$-subdivision in $G$. In other words, $G$ is $H$-linked if $G$ contains an $H$ subdivision with prescribed branching vertices for any such prescription. This notion is a common generalization of the notions of $k$-linked, $k$-ordered, and $k$-connected graphs. In particular, if $G$ is $k$-linked, then $G$ is $H$-linked for every $H$ with $k$ edges and no isolated vertices. Since every $10 k$-connected graph is $k$-linked (see [8]), every $10(|E(H)|+|V(H)|)$-connected graph is $H$-linked.

The idea of $H$-linked graphs originated with Jung [3], but had not been considered in full generality until recently, when the concept was first considered independently in $[5]$ and $[10]$.

In [6] and [7], $H$-linkage was considered for loopless multigraphs $H$ with $k$ edges and minimum degree at least two. The following was shown in [7].

TheOrem 1. Let $H$ be a loopless graph with $|E(H)|=k$ and $\delta(H) \geq 2$. Every simple graph $G$ of order $n \geq 5 k+6$ with $\delta(G) \geq\left\lceil\frac{n+k}{2}\right\rceil-1$ is $H$-linked. If $H=C_{k}$,

\footnotetext{
* Received by the editors February 17, 2005; accepted for publication (in revised form) March 6, 2006; published electronically December 5, 2006.

http://www.siam.org/journals/sidma/20-4/62466.html

${ }^{\dagger}$ Department of Mathematics and Computer Science, Emory University, Atlanta, GA 30322 (rg@mathcs.emory.edu).

${ }^{\ddagger}$ Department of Mathematics, University of Illinois, Urbana, IL 61801 and Institute of Mathematics, Novosibirsk, 630090, Russia (kostochk@math.uiuc.edu). This author was partially supported by NSF grant DMS-0400498 and grant 03-01-00796 of the Russian Foundation for Basic Research.

$\S$ Department of Mathematics, University of Illinois, Urbana, IL 61801 (gexinyu@uiuc.edu).
} 
then every graph $G$ of order $n \geq 5 k+6$ with $\delta(G) \geq\left\lceil\frac{n}{2}\right\rceil+\left\lfloor\frac{k}{2}\right\rfloor-1$ is $H$-linked. The minimum degree conditions are sharp.

It was also verified in [7] that under the conditions of Theorem 1, any $H$-subdivision in $G$ can be extended to an $H$-subdivision that spans $V(G)$. This extended an earlier result of Kierstead, Sárközy, and Selkow [4] on $k$-ordered graphs. Extension results like this and that of [2] provide a framework for generalizing both linkage and strong Hamiltonian-type results, as both involve questions on spanning subgraphs.

In [6], the work in [7] was sharpened. At the same time a result similar to that of [6] was shown in [1].

Let $B(H)$ denote the maximum number of edges in an edge-cut of $H$. In terms of $B(H)$, the main results in [6] and [1] can be summarized as follows.

THEOREM 2. Every simple graph $G$ of order $n$ with $\delta(G) \geq\left\lceil\frac{n+B(H)}{2}\right\rceil-1$ is $H$-linked provided

(i) See [6]. $H$ is a loopless connected multigraph with $k$ edges and $\delta(H) \geq 2$ and $G$ is of order $n \geq 7.5 k$.

(ii) See [1]. $H$ is a connected multigraph, possibly containing loops, and $G$ is of sufficiently large order $n$.

The purpose of this paper is to provide a merging of ideas from [6] and [1] and to prove a more general result describing the situations also for disconnected graphs $H$. That is, we wish to show for all multigraphs $H$, possibly containing loops, a sharp lower bound on $\delta(G)$ sufficient to ensure that each graph $G$ on at least $10(|V(H)|+|E(H)|)$ vertices will be $H$-linked. It turns out that for disconnected $H$, the bound is more sophisticated.

We will say that a multigraph $H$ is uneven if it does not contain even cycles. Denote by $c(H)$ the number of uneven components of $H$. Let

$$
b(H)= \begin{cases}|V(H)|-1 & \text { if } H \text { is uneven } \\ B(H)+c(H) & \text { otherwise. }\end{cases}
$$

Note that for uneven graphs, the value $b(H)=|V(H)|-1$ is exactly one less than that from the second part of the formula.

Our proof is based on the proof in [6], modified to handle the more general conditions on $H$. Our main result is the following theorem.

TheOREM 3. Let $H$ be a multigraph with $e(H)$ edges (loops or nonloops) and let $k_{1}=k_{1}(H)=e(H)+c(H)$. Let $G$ be a simple graph of order $n \geq 9.5\left(k_{1}+1\right)$. If

$$
\delta(G) \geq\left\lceil\frac{n+b(H)}{2}\right\rceil-1
$$

then $G$ is $H$-linked. Moreover, every injective mapping $f: V(H) \rightarrow V(G)$ can be extended to an $H$-subdivision in $G$ containing at most $5 k_{1}+2$ vertices.

Restriction (1.1) cannot be weakened. In the next section we will prove this and derive some simple facts on edge cuts in connected graphs. In the subsequent three sections we prove Theorem 3 for the case of loopless $H$, and in the final section we prove the theorem in full generality. We briefly discuss the ideas of the proof at the end of section 3 .

2. On edge cuts and constructions. It is well known (see, e.g., [9, p. 51]) that

$$
B(H) \geq(k+1) / 2
$$


for every $H$ with $k>0$ edges.

The following property makes uneven components special for our theorem.

Lemma 4. A connected graph $H$ is uneven if and only if $B(H)=|V(H)|-1$.

Proof. Suppose first that $H$ is a connected uneven graph with $m$ cycles. Then no two cycles in $H$ share an edge and hence $|E(H)|=|V(H)|-1+m$. Furthermore, any edge cut in $H$ misses at least one edge in each (odd) cycle of $H$, and hence $B(H) \leq|E(H)|-m$. Therefore $B(H) \leq|V(H)|-1$. On the other hand, if we delete one edge from each (odd) cycle of $H$, then we obtain a bipartite graph. Hence $B(H)=|V(H)|-1$.

Suppose now that a connected graph $H$ contains an even cycle $C=\left(w_{1}, \ldots, w_{2 m}\right)$. Let $V_{1}=\left\{w_{1}, w_{3}, \ldots, w_{2 m-1}\right\}$ and $V_{2}=\left\{w_{2}, w_{4}, \ldots, w_{2 m}\right\}$. Then at least $\left|V_{1}\right|+\left|V_{2}\right|$ edges in $H$ connect $\left|V_{1}\right|$ with $\left|V_{2}\right|$. If $V_{1} \cup V_{2}=V(G)$, then we have $B(H) \geq|V(H)|$. Otherwise, since $H$ is connected, there is a vertex $w_{2 m+1}$ adjacent to $V_{1} \cup V_{2}$. If $w_{2 m+1}$ is adjacent to $V_{1}$, then we add it to $V_{2}$, otherwise add it to $V_{1}$. In any case, the number of edges between the new $V_{1}$ and $V_{2}$ is greater than between the old ones. We continue adding vertices to $V_{1} \cup V_{2}$ so that with each added vertex, the number of edges between $V_{1}$ and $V_{2}$ grows by at least one. When we add the last vertex of $H$, we get a partition $\left(V_{1}, V_{2}\right)$ of $V(H)$ such that the number of edges between $V_{1}$ and $V_{2}$ is at least $|V(H)|$.

Now we show that restriction (1.1) in Theorem 3 cannot be weakened.

Suppose first that the multigraph $H$ has no uneven components. In this case, by definition, $b(H)=B(H)=\max _{X \subset V(H)} e(X, V(H)-X)$. Let this maximum be achieved at the set $X_{0} \subset V(H)$ and let $Y_{0}=V(H)-X_{0}$. Let $G$ be formed from two complete graphs $G_{1}$ and $G_{2}$ of order $l$ that intersect on $b(H)-1$ vertices. If the set $S$ chosen as the image of $V(H)$ under $f$ is such that the vertices of $X_{0}$ lie in $G_{1}-G_{2}$ and the vertices of $Y_{0}$ lie in $G_{2}-G_{1}$, then $G_{1} \cap G_{2}$ is not large enough to allow an embedding of $H$. Further, $\delta(G)=l-1$. Since $|V(G)|=2 l-b(H)+1$, we see that $\delta(G)=\frac{n+b(H)-3}{2}$. Thus, (1.1) is necessary in this case.

Suppose now that $H$ has both uneven components and components containing even cycles. Let $H_{0}$ be the subgraph of $H$ induced by all uneven components of $H$ and $H_{1}$ be the subgraph of $H$ induced by all other components. By our definition and Lemma 4 ,

$$
b(H)=B\left(H_{1}\right)+\left|V\left(H_{0}\right)\right| .
$$

Let $X_{1}$ be a subset of $V\left(H_{1}\right)$ such that $\left(X_{1}, V\left(H_{1}\right)-X_{1}\right)$ is a maximum edge cut and let $Y_{1}=V\left(H_{1}\right)-X_{1}$. Then $B\left(H_{1}\right)=e\left(X_{1}, Y_{1}\right)$. Consider the same graph $G$ as the previous paragraph. Let the mapping $f$ be such that the image of $X_{1}$ is completely in $G_{1}-G_{2}$, the image of $Y_{1}$ is completely in $G_{2}-G_{1}$, and the image of $V\left(H_{0}\right)$ is completely in $G_{1} \cap G_{2}$. Then only $b(H)-1-\left|V\left(H_{0}\right)\right|$ vertices of $G_{1} \cap G_{2}$ are not occupied by vertices of $H_{0}$. By (2.2), this is not enough to embed all paths from the image of $X_{1}$ to the image of $Y_{1}$.

If every component of $H$ is uneven, we will map the vertices of all but one component, say $C_{0}$, from $H$ to $G_{1} \cap G_{2}$ and then place the vertices of $C_{0}$ into $G_{1}-G_{2}$ and $G_{2}-G_{1}$ so that we need $\left|V\left(C_{0}\right)\right|-1$ paths to connect $G_{1}-G_{2}$ with $G_{2}-G_{1}$.

3. Preliminaries. In this and the next two sections we consider only loopless $H$. First, for the purposes of our proof, we wish to show that it suffices to consider only $H$ with no uneven components, or $H$ that are connected and contain an odd cycle, or $H=K_{2}$. Note that if $H^{\prime}$ is obtained from $H$ by adding an edge $e^{\prime}$ and if $k_{1}\left(H^{\prime}\right) \leq k_{1}(H)$ and $b\left(H^{\prime}\right) \leq b(H)$, then, since $H^{\prime} \supset H$, the fact that a graph $G$ is 
$H^{\prime}$-linked implies that $G$ is $H$-linked. Now, if $H$ has at least two components and a component $H_{1}$ of $H$ is uneven, then by adding an edge connecting $H_{1}$ with another component, we decrease $c(H)$. This means that $b(H)$ and $k_{1}(H)$ do not change. Thus, in this case it is enough to consider only the cases when $H$ is connected or has no uneven components. Furthermore, if $H$ is a tree on at least 3 vertices, then adding to $H$ an edge connecting two vertices at distance two does not change $c(H)$ or $b(H)$, but now $H$ contains an odd cycle. If $H$ is a tree on 2 vertices, then $H=K_{2}$ and hence $b(H)=1$. Thus, it suffices to consider the case when $H$ has no uneven components, or $H$ is a connected graph containing an odd cycle, or $H=K_{2}$, and the reduction we desired is possible.

Suppose that $e(H)=k$. Let $f: V(H) \rightarrow V(G)$ be an injective mapping and $W=f(V(H))$. Let $E(H)=\left\{e_{j}=u_{j}^{0} v_{j}^{0}: 1 \leq j \leq k\right\}$. Let $u_{j}=f\left(u_{j}^{0}\right)$ and $v_{j}=f\left(v_{j}^{0}\right)$.

If $H=K_{2}$, then $k=1$ and $b(H)=1$. In this case, if an $n$-vertex graph $G$ satisfies the conditions of the theorem, then $\delta(G) \geq(n-1) / 2$. Therefore $u_{1}$ and $v_{1}$ are either adjacent or have a common neighbor. This settles the case of $H=K_{2}$, and from now on we assume that either $H$ is connected and has a cycle or has no uneven components. In this case, $|W|=|V(H)| \leq k$.

For each edge $e_{j}=u_{j}^{0} v_{j}^{0} \in E(H)$, we define functions $\beta\left(e_{j}, u_{j}^{0}\right), \beta\left(e_{j}, v_{j}^{0}\right)$ inductively as follows:

(1) If $H$ has no vertices of degree one, then for every $j$, let $\beta\left(e_{j}, u_{j}^{0}\right)=1 / \operatorname{deg}_{H}\left(u_{j}^{0}\right)$ and $\beta\left(e_{j}, v_{j}^{0}\right)=1 / \operatorname{deg}_{H}\left(v_{j}^{0}\right)$.

(2) If $H$ has a pendant vertex $u_{s}^{0}$ (which is incident with the edge $e_{s}=u_{s}^{0} v_{s}^{0}$ ), let $H^{\prime}=H-u_{s}^{0}$. Since $H^{\prime}$ is a smaller graph without acyclic components, we can define $\beta\left(e_{j}, u_{j}^{0}\right), \beta\left(e_{j}, v_{j}^{0}\right)$ for every $j \neq s$ and then let $\beta\left(e_{s}, u_{s}^{0}\right)=1$ and $\beta\left(e_{s}, v_{s}^{0}\right)=0$.

For simplicity, we denote $\beta\left(e_{j}, u_{j}^{0}\right)$ by $\beta_{j}$, and $\beta\left(e_{j}, v_{j}^{0}\right)$ by $\gamma_{j}$. By construction, for every $j=1, \ldots, k$,

$$
0 \leq \beta_{j}, \gamma_{j} \leq 1 \text { and } \beta_{j}+\gamma_{j} \leq 1
$$

Also, for every $u^{0} \in V(H)$,

$$
\sum_{\left\{e \in E(H): u^{0} \in e\right\}} \beta\left(e, u^{0}\right)=1, \quad \text { and hence } \sum_{j=1}^{k}\left(\beta_{j}+\gamma_{j}\right)=|V(H)|=|W| .
$$

Say that a family $\mathcal{C}$ of the form $\left\{P_{1}, \ldots, P_{k}\right\}$ is a partial $H$-linkage if each $P_{j}$ is either the set $\left\{u_{j}, v_{j}\right\}$ or a $u_{j}, v_{j}$-path and the following conditions hold:

(I) $|X| \leq|W|+3 k-2 b(H)+2 \alpha+3$, where $X=\bigcup_{j=1}^{k} V\left(P_{j}\right)$ and $\alpha$ is the number of $P_{j}$-s that are paths;

(II) The internal vertices of the paths $P_{j}$ 's are pairwise disjoint and disjoint from $W$.

Consider $\mathcal{C}_{0}=\left\{\left\{u_{1}, v_{1}\right\}, \ldots,\left\{u_{k}, v_{k}\right\}\right\}$. This family satisfies the properties (I) and (II) above with $X=\bigcup_{j=1}^{k}\left\{u_{j}, v_{j}\right\}=W$ and $\alpha=0$. Therefore, $\mathcal{C}_{0}$ is a partial $H$-linkage.

A partial $H$-linkage $\mathcal{C}=\left\{P_{1}, \ldots, P_{k}\right\}$ is optimal, if as many $P_{j}$-s as possible are paths and, subject to this, the set $X=\bigcup_{j=1}^{k} V\left(P_{j}\right)$ is as small as possible. We will prove that an optimal partial $H$-linkage is an $H$-subdivision. This will imply our theorem (for loopless $H$ ).

Suppose, to the contrary, that $\mathcal{C}=\left\{P_{1}, \ldots, P_{k}\right\}$ is an optimal partial $H$-linkage but is not an $H$-subdivision. Let, for definiteness, $P_{k}=\left\{u_{k}, v_{k}\right\}$ and $u_{k} v_{k} \notin E(G)$. 
Denote $X=\bigcup_{j=1}^{k} V\left(P_{j}\right), x=u_{k}$, and $y=v_{k}$. Let $A=N(x)-X, B=N(y)-X$, and $R=V(G)-(X \cup A \cup B)$.

By (1.1) and (2.1), each of $A$ and $B$ has size at least

$$
\begin{aligned}
\delta(G)-(|X|-2) & \geq \frac{n+b(H)-2}{2}-(|W|+3 k-2 b(H)+2(k-1)+3-2) \\
& \geq \frac{9.5 k+b(H)-2}{2}-6 k+1+2 b(H)=2.5 b(H)-1.25 k \geq 1.25 .
\end{aligned}
$$

It follows that we may choose distinct $a_{1}, a_{2} \in A$ and $b_{1}, b_{2} \in B$.

For $v \in V(G)$, let $d_{j}(v)$ denote the number of neighbors of $v$ in the interior of $P_{j}$ plus $\beta_{j}$ if $u_{j} \in N_{G}(v)$ and plus $\gamma_{j}$ if $v_{j} \in N_{G}(v)\left(\beta_{j}\right.$ and $\gamma_{j}$ are defined above (3.1)). By (3.2), we have

$$
\sum_{j=1}^{k} d_{j}(v)=\left|N_{G}(v) \cap X\right| \quad \forall v \in V(G) .
$$

Let $l_{p}$ be the number of $P_{j}$ 's of length $p$ for $p \geq 1$, and $l_{0}$ be the number of $P_{j}$ 's that are not paths. Then

$$
|X|=|W|+\sum_{p \geq 1}(p-1) l_{p}=\sum_{j=1}^{k}\left(\beta_{j}+\gamma_{j}\right)+\sum_{p \geq 1}(p-1) l_{p}
$$

and

$$
k=\sum_{p \geq 0} l_{p}=\alpha+l_{0} .
$$

We will assume that every path $P_{j}$ is of the form $P_{j}=u_{j}, w_{1, j}, \ldots, w_{p_{j}-1, j}, v_{j}$. Sometimes, for simplicity we will write $p$ instead of $p_{j}$ and $w_{i}$ instead of $w_{i, j}$ if $j$ is clear from the context. In the rest of the paper, for every $j=1, \ldots, k$ and fixed $a_{1}, a_{2} \in A$, $b_{1}, b_{2} \in B$, we denote $M_{j}=d_{j}(x)+d_{j}(y)$ and $L_{j}=d_{j}\left(a_{1}\right)+d_{j}\left(a_{2}\right)+d_{j}\left(b_{1}\right)+d_{j}\left(b_{2}\right)$.

In order to add an $x, y$-path to $\mathcal{C}$ and still satisfy condition (I), we are allowed to use only two additional vertices. In the next section, we prove that, for an optimal $\mathcal{C}$, the set $X$ satisfies an inequality stronger than (I) and this allows us to use five additional vertices when constructing an $x, y$-path. We will eventually show that if even with the help of that many vertices we are not able to create an $x, y$-path, possibly changing already constructed paths, then either $x$ or $y$ has a low degree.

4. Main lemma. We begin with a lemma needed in the proof of Lemma 6 .

Lemma 5. Let $a_{1}, a_{2} \in A, b_{1}, b_{2} \in B$. For $a P_{j}=u_{j}, w_{1}, \ldots, w_{p-1}, v_{j}$, let $s_{j}=M_{j}+0.5 L_{j}, \beta=\beta_{j}$, and $\gamma=\gamma_{j}$. Define

$$
D_{1}(p, \beta, \gamma)= \begin{cases}p+2+2 \beta+2 \gamma & \text { for } p \leq 1 \\ p+4+2 \beta+2 \gamma & \text { for } p \geq 2 .\end{cases}
$$

Then

(a) $s_{j} \leq D_{1}(p, \beta, \gamma)$.

(b) $s_{k} \leq 2\left(\beta_{k}+\gamma_{k}\right)$. Furthermore, if $x y=u_{k} v_{k} \notin E(G)$, then $s_{k}=\beta_{k}+\gamma_{k}$. 
Proof. Let $\lambda=\max \{\beta, \gamma\}$. By definition (see (3.1)), $\lambda \leq 1, \min \{\beta, \gamma\} \leq 0.5$, and $L_{k}=2 \beta_{k}+2 \gamma_{k}$. If $x y \in E(G)$, then $M_{k}=\beta_{k}+\gamma_{k}$; otherwise, $M_{k}=0$. This proves (b).

Claim 1. Let $Z=\left\{a_{1}, a_{2}, b_{1}, b_{2}\right\}$.

(i) For each $z \in Z$, the distance in $P_{j}$ between any two neighbors of $z$ is at most two. In particular, each $z \in Z$ has at most 3 neighbors in $P_{j}$.

(ii) If $p \geq 3$, then no $z \in Z$ is a common neighbor of $u_{j}$ and $v_{j}$.

(iii) If $p \geq 3$, then $x$ and $y$ have no interior neighbors of distance at most $p-3$ in $P_{j}$.

(iv) If $p \geq 3$, then $x$ (respectively, $y$ ) has no interior neighbors at distance at most $p-4$ in $P_{j}$ from interior neighbors of $b_{1}$ and $b_{2}$ (respectively, of $a_{1}$ and $a_{2}$ ).

Proof. If some $z \in Z$ is adjacent to $w_{i}$ and $w_{i+m}$ for some $m \geq 3$ (we treat $u_{j}$ as $w_{0}$ and $v_{j}$ as $w_{p}$ ), then we can replace $P_{j}$ by a shorter $u_{j}, v_{j}$-path, a contradiction to the optimality of $\mathcal{C}$. This proves (i), and (ii) is a partial case of (i).

If $x$ and $y$ have interior neighbors at distance at most $p-3$ in $P_{j}$, then we can delete $P_{j}$ from $\mathcal{C}$ and add a shorter $x, y$-path. This proves (iii). The same trick proves (iv), completing the proof of the claim.

In order to prove (a), we consider several cases (depending on $p$ ).

Case 1. $p=0$. By $(3.1), L_{j} \leq 4(\beta+\gamma) \leq 4$. Therefore $s_{j}=M_{j}+0.5 L_{j} \leq$ $2(\beta+\gamma)+2=D_{1}(0, \beta, \gamma)$.

Case 2. $p=1$. Trivially,

$$
s_{j} \leq 2(\beta+\gamma)+0.5(4(\beta+\gamma)) \leq 2(\beta+\gamma)+2<D_{1}(1, \beta, \gamma) .
$$

Case 3. $p=2$. If each of $x$ and $y$ is adjacent to $w_{1}$ and some $z \in Z$ is adjacent to both $u_{j}$ and $v_{j}$, then $\mathcal{C}$ is not optimal: we can replace $P_{j}$ by the path $u_{j}, z, v_{j}$ and add the path $x w_{1} y$. Otherwise, either $M_{j} \leq 2(\beta+\gamma)+1$ and hence

$$
s_{j} \leq 2(\beta+\gamma)+1+0.5(4(\beta+\gamma+1)) \leq 2(\beta+\gamma)+6=D_{1}(2, \beta, \gamma),
$$

or $L_{j} \leq 4(\lambda+1)$ and hence

$$
s_{j} \leq 2(\beta+\gamma+1)+0.5(4(\lambda+1)) \leq 2(\beta+\gamma)+6=D_{1}(2, \beta, \gamma) .
$$

Case 4. $p=3$. By (iii), $M_{j} \leq 2(\beta+\gamma)+2$. If $L_{j} \leq 10$, then $s_{j} \leq D_{1}(3, \beta, \gamma)$. Otherwise, because of the symmetry between $A$ and $B$, we may assume that $d_{j}\left(a_{1}\right)+$ $d_{j}\left(a_{2}\right)>5$ and that $d_{j}\left(a_{1}\right)>2.5$. Then by (ii), we may assume that $a_{1}$ is adjacent to $w_{1}, w_{2}$, and $v_{j}$ and that $a_{2}$ is adjacent to $w_{1}$ and $w_{2}$ (and maybe to one more vertex). If $y w_{2} \in E(G)$, then we can replace $P_{j}$ with $u_{j}, w_{1}, a_{1}, v_{j}$ and add the path $x, a_{2}, w_{2}, y$, a contradiction to the optimality of $\mathcal{C}$. If neither $x$ nor $y$ is adjacent to $w_{2}$, then by (iii), $M_{j} \leq 2(\beta+\gamma)+1$, by (ii), $L_{j} \leq 4(2+\lambda) \leq 12$, and therefore $s_{j} \leq 2(\beta+\gamma)+7=D_{1}(3, \beta, \gamma)$. If $x w_{2} \in E(G)$ and some $b \in\left\{b_{1}, b_{2}\right\}$ is adjacent to $w_{2}$, then we can replace $P_{j}$ with $u_{j}, w_{1}, a_{1}, v_{j}$ and add the path $x, w_{2}, b, y$. Finally, if neither $b_{1} w_{2}$ nor $b_{2} w_{2}$ is in $E(G)$, then by (i), $d_{j}\left(b_{1}\right)+d_{j}\left(b_{2}\right) \leq 2(1+\lambda) \leq 4$, and hence by (ii) $L_{j} \leq 6+4=10$.

Case 5. $p \geq 4$. If $x$ has $r$ interior neighbors and $r \geq 2$, then by (iii), $d_{j}(y) \leq \beta+\gamma$ and by (iv), $d_{j}\left(b_{i}\right) \leq \max \{0,3-r\}+\lambda$. Together with (i) this shows that in this case,

$$
s_{j} \leq 2 \beta+2 \gamma+r+3+\max \{0,3-r\}+\lambda .
$$

If $r \geq 3$, then $s_{j} \leq 2 \beta+2 \gamma+p-1+3+\lambda \leq p+3+2 \beta+2 \gamma \leq D_{1}(p, \beta, \gamma)$. If $r=2$, then $s_{j} \leq 2 \beta+2 \gamma+r+4+\lambda \leq 2 \beta+2 \gamma+p+3 \leq D_{1}(p, \beta, \gamma)$, again. 
Thus, we can assume that each of $x$ and $y$ has at most one interior neighbor in $P_{j}$. By (iv) $d_{j}\left(a_{i}\right)+d_{j}(y) \leq \beta+\gamma+\lambda+3$ and $d_{j}\left(b_{i}\right)+d_{j}(x) \leq \beta+\gamma+\lambda+3$ for $i=1,2$. Therefore, $s_{j} \leq 2 \lambda+6+2 \beta+2 \gamma \leq 2 \beta+2 \gamma+p+2+2=D_{1}(p, \beta, \gamma)$. This completes the proof of (a) and hence, of Lemma $5 . \quad \square$

Lemma 6. Let $a_{1}, a_{2} \in A, b_{1}, b_{2} \in B, Z=\left\{a_{1}, a_{2}, b_{1}, b_{2}\right\}$, and $V_{0}=(A \cup B)-$ $Z-N_{G}(Z)$. Then $|X| \leq|W|+3 k-2 b(H)+2 \alpha-|R|-\left|V_{0}\right|$.

Proof. Let

$$
\Sigma^{\prime}=\operatorname{deg}_{G}(x)+\operatorname{deg}_{G}(y)+\frac{1}{2}\left(\operatorname{deg}_{G}\left(a_{1}\right)+\operatorname{deg}_{G}\left(a_{2}\right)+\operatorname{deg}_{G}\left(b_{1}\right)+\operatorname{deg}_{G}\left(b_{2}\right)\right) .
$$

Observe that every vertex $w \notin X$ contributes to $\Sigma^{\prime}$ at most 2 : if $w \in R$, then it is not adjacent to $x$ and $y$, and if $w \in A$ (respectively, $w \in B$ ), then it is not adjacent to $y, b_{1}$, and $b_{2}$ (respectively, to $x, a_{1}$, and $a_{2}$ ). By definition, every vertex in $V_{0}$ is not adjacent to any vertex in $Z$, and therefore contributes at most 1 to $\Sigma^{\prime}$. Furthermore, every $z \in Z$ contributes at most 1.5 to $\Sigma^{\prime}$, since it is not adjacent to itself. Therefore,

$$
\Sigma^{\prime} \leq 4 \cdot 1.5+2(|A \cup B|-4)+2|R|+\sum_{j=1}^{k} s_{j}-\left|V_{0}\right| .
$$

By Lemma 5, (3.2), and (3.5),

$$
\begin{aligned}
\sum_{j=1}^{k} s_{j} & \leq k+l_{0}+2 l_{1}+\sum_{p \geq 2}(p+3) l_{p}+2 \sum_{j=1}^{k}\left(\beta_{j}+\gamma_{j}\right)-1 \\
& =k+l_{0}+2 l_{1}+\sum_{p \geq 2}(p+3) l_{p}+2|W|-1
\end{aligned}
$$

Therefore,

$$
\begin{aligned}
\Sigma^{\prime} \leq & 2(|A \cup B|+|R|)-2-\left|V_{0}\right|+2\left(|W|+l_{0}+\sum_{p \geq 1} p l_{p}\right) \\
& -1-l_{0}+\sum_{p \geq 2}(3-p) l_{p}+k .
\end{aligned}
$$

By (3.4) and (3.5), the last expression is equal to $2 n+3 k-\left|V_{0}\right|-3-l_{0}-\sum_{p \geq 2}(p-3) l_{p}$. Combining this again with (3.4) and (3.5), we get

$$
|X|+\Sigma^{\prime} \leq 2 n+|W|+3 k+2 \alpha-3-l_{0}-2 l_{1}-\left|V_{0}\right| .
$$
Thus,

By the assumption of Theorem $3, \delta(G) \geq \frac{n+b(H)}{2}-1$ and hence $\Sigma^{\prime} \geq 2 n+2 b(H)-4$.

$$
\begin{aligned}
|X| & \leq|W|+3 k-2 b(H)+2 \alpha-l_{0}-2 l_{1}-\left|V_{0}\right|+1 \\
& \leq|W|+3 k-2 b(H)+2 \alpha-\left|V_{0}\right| .
\end{aligned}
$$

If an $r \in R$ has a neighbor $a_{0} \in A$ and a neighbor $b_{0} \in B$, then one can add to $\mathcal{C}$ the path $P_{k}=x, a_{0}, r, b_{0}, y$. The new set of paths will be a better partial linkage, since the new $X$ would have size at most $|W|+3 k-2 b(H)+2(\alpha+1)+1$. Since this 
contradicts the choice of $\mathcal{C}$, no $r \in R$ has both a neighbor in $A$ and a neighbor in $B$. Thus every $r \in R$ contributes at most 1 to $\Sigma^{\prime}$, and (4.2) becomes

$$
\Sigma^{\prime} \leq 4 \cdot 1.5+2(|A \cup B|-4)+|R|+\sum_{j=1}^{k} s_{j}-\left|V_{0}\right| .
$$

Correspondingly, (4.4) transforms into

$$
|X| \leq|W|+3 k-2 b(H)+2 \alpha-\left|V_{0}\right|-|R| .
$$

5. Completion of the case of loopless $\boldsymbol{H}$. Lemma 6 has the following two immediate consequences.

Lemma 7. $|A|+|B|>2 k$.

Proof. By Lemma 6 and $(2.1),|A|+|B|=n-(|X|+|R|) \geq n-(|W|+3 k-$ $2 b(H)+2 \alpha) \geq 9.5 k-\left(k+3 k-2 \frac{k+1}{2}+2(k-1)\right)=4.5 k+3>2 k$.

Lemma 8. Each $v \in V(G)$ is adjacent to at least 3 vertices in $A \cup B-V_{0}$. In particular, either $v$ has 2 neighbors in $A$ that belong to or are adjacent to the set $\left\{a_{1}, a_{2}\right\}$, or 2 neighbors in $B$ that belong to or are adjacent to the set $\left\{b_{1}, b_{2}\right\}$.

Proof. Recall that by the definition of $V_{0}, A \cup B-V_{0}=Z \cup\left(N_{G}(Z) \cap(A \cup B)\right)$. Hence, by Lemma 6 ,

$$
\begin{aligned}
\delta(G)-\left(|X|+|R|+\left|V_{0}\right|\right) & \geq 0.5(9.5 k+b(H)-2)-|W|-3 k+2 b(H)-2 \alpha \\
& \geq 4.75 k+0.5 b(H)-1-k-3 k+2 b(H)-2(k-1) \\
& =2.5 b(H)-1.25 k+1 \geq 2.25>2 .
\end{aligned}
$$

Thus each vertex has at least 3 neighbors in $V(G)-X-R-V_{0}=A \cup B-V_{0}$.

For given $a_{1}, a_{2} \in A, b_{1}, b_{2} \in B$, let $A^{\prime \prime}=A^{\prime \prime}\left(a_{1}, a_{2}\right)$ (respectively, $B^{\prime \prime}=$ $\left.B^{\prime \prime}\left(b_{1}, b_{2}\right)\right)$ denote the set of vertices in $X$ having at least 2 neighbors in $A$ (respectively, in $B$ ) that belong to or are adjacent to the set $\left\{a_{1}, a_{2}\right\}$ (respectively, $\left\{b_{1}, b_{2}\right\}$ ). The above lemma yields that for every choice of $a_{1}, a_{2}, b_{1}$, and $b_{2}$,

$$
A^{\prime \prime} \cup B^{\prime \prime}=X \text {. }
$$

Lemma 9. For every nonadjacent $s, t \in A$ (or $B$ ), $|N(s) \cap N(t)-X| \geq 3$.

Proof. Suppose to the contrary that $a_{1}, a_{2} \in A, a_{1} a_{2} \notin E(G)$ and the cardinality of the set $T$ of common neighbors of $a_{1}$ and $a_{2}$ outside of $X$ is at most two. Consider arbitrary $b_{1}, b_{2} \in B$ and let $Z=\left\{a_{1}, a_{2}, b_{1}, b_{2}\right\}$. Then the contribution of every $a \in A-Z-T$ to the sum $\Sigma^{\prime}$ defined in (4.1) is at most 1.5. Thus, repeating the proof of Lemma 6 , the right-hand side of the inequality corresponding to (4.5) will be less by $0.5|A-Z-T|$. Hence, since $|(Z \cap A) \cup T| \leq 4$, instead of (4.5), we will get $|X| \leq|W|-|R|+3 k-2 b(H)+2 \alpha-\left|V_{0}\right|-0.5\left(\left|A-V_{0}\right|-4\right)$. In other words,

$$
|X|+0.5|A|+|R| \leq|W|+3 k-2 b(H)+2 \alpha+2 \leq 6 k-2 b(H) .
$$

On the other hand, $\operatorname{deg}_{G-X}\left(a_{1}\right)+\operatorname{deg}_{G-X}\left(a_{2}\right) \leq|A|+|T|+|R|-2$ (the -2 arises because neither $a_{1}$ nor $a_{2}$ is adjacent to $a_{1}$ or $a_{2}$ ). It follows that

$$
2 \frac{n+b(H)}{2}-2 \leq 2 \delta(G) \leq 2|X|+|A|+|R|,
$$


which together with (5.2) yields $n+b(H)-2 \leq 2(6 k-2 b(H))$. Thus, $n \leq 12 k-$ $5 b(H)+2 \leq 12 k-5 \frac{k+1}{2}+2=9.5 k-0.5$, a contradiction.

For the rest of the section, we fix some distinct $a_{1}, a_{2} \in A$ and $b_{1}, b_{2} \in B$, and let $A^{\prime \prime}=A^{\prime \prime}\left(a_{1}, a_{2}\right)$ and $B^{\prime \prime}=B^{\prime \prime}\left(b_{1}, b_{2}\right)$.

Lemma 10. Let $\mathcal{C}$ be optimal, $1 \leq j \leq k-1$, and either $\left\{u_{j}, v_{j}\right\} \subset A^{\prime \prime}$ or $\left\{u_{j}, v_{j}\right\} \subset B^{\prime \prime}$. Then for each $a \in A$ and $b \in B$,

$$
\left(N(a) \cap N(b) \cap P_{j}\right) \backslash\left\{u_{j}, v_{j}\right\}=\emptyset .
$$

Proof. Assume to the contrary that $r \in N(a) \cap N(b) \cap P_{j} \backslash\left\{u_{j}, v_{j}\right\}$. Let $P_{k}^{\prime}=$ $(x, a, r, b, y)$. Without loss of generality, assume that $\left\{u_{j}, v_{j}\right\} \subset A^{\prime \prime}$. Then there exist $s \in N\left(u_{j}\right) \cap A \backslash\{a\}$ and $t \in N\left(v_{j}\right) \cap A \backslash\{a\}$. If $s=t$ or $s$ is adjacent to $t$, then let $P_{j}^{\prime}=\left(u_{j}, s, t, v_{j}\right)$.

If $s$ and $t$ are nonadjacent, then by Lemma 9 , we have $|(N(s) \cap N(t)) \backslash X| \geq 3$, and therefore there exists $q \in N(s) \cap N(t) \backslash(X \cup\{a, b\})$. In this case, let $P_{j}^{\prime}=\left(u_{j}, s, q, t, v_{j}\right)$. In both cases, $P_{j}^{\prime}$ is a path disjoint from $P_{k}^{\prime}$. Thus, in both cases we increase the number of $P_{j}$-s that are paths by one and, by (4.5), maintain $|X| \leq|W|+3 k-$ $2 b(H)+2(\alpha+1)+3$. This is a contradiction which completes the proof.

Lemma 11. Let $\mathcal{C}$ be optimal, $1 \leq j \leq k-1, P_{j}=\left(w_{0}, w_{1}, \ldots, w_{p}\right)$, where $w_{0}=u_{j} \in A^{\prime \prime}$, and $w_{p}=v_{j} \in B^{\prime \prime}$. If some $w_{i}, 1 \leq i \leq p-1$, has a neighbor $a_{0} \in A \cup\{x\}$ and a neighbor $b_{0} \in B \cup\{y\}$, then each $w_{i^{\prime}}$ for $i<i^{\prime} \leq p$ has no neighbors in $A-a_{0}$ and each $w_{i^{\prime \prime}}$ for $0 \leq i^{\prime \prime}<i$ has no neighbors in $B-b_{0}$.

Proof. Suppose some $w_{i^{\prime}}$ for $i<i^{\prime} \leq p$ has a neighbor $a^{\prime} \in A-a_{0}$. By the definition of $A^{\prime \prime}, u_{j}$ has a neighbor $a^{\prime \prime} \in A-a_{0}$. By Lemma 9 , the length of a shortest path $P^{\prime}$ from $a^{\prime \prime}$ to $a^{\prime}$ in $G\left[A-a_{0}\right]$ is at most two. Thus, we can replace $P_{j}$ by the path $\left(u_{j}, a^{\prime \prime}, P^{\prime}, a^{\prime}, w_{i^{\prime}}, P_{j}^{\prime}, v_{j}\right.$ ) (where $P_{j}^{\prime}$ is the part of $P_{j}$ connecting $w_{i^{\prime}}$ with $v_{j}$ ) and add the path $P_{k}=\left(x, a_{0}, w_{i}, b_{0}, y\right)$. The new set of $\alpha+1$ paths has at most $|X|+5$ vertices, which by (4.5) is at most $|W|+3 k-2 b(H)+2(\alpha+1)+3$, a contradiction to the choice of $\mathcal{C}$. Note that a similar argument works for $w_{i^{\prime \prime}}$.

Similarly to $d_{j}(v)$, let $d_{j}(u, v)$ denote the number of common neighbors of $u$ and $v$ "inside" $P_{j}$ plus $\beta_{j} \cdot\left|N(u) \cap N(v) \cap\left\{u_{j}\right\}\right|$ plus $\gamma_{j} \cdot\left|N(u) \cap N(v) \cap\left\{v_{j}\right\}\right|$.

Lemma 12. Let $\mathcal{C}$ be optimal, $a \in A, b \in B$. Then there exists some $j=j(a, b)$ such that $d_{j}(a, b)>1$.

Proof. Since $N(a) \cap N(b) \cap(V(G)-X+x+y)=\emptyset$ (otherwise we can find a path xazby not using any vertex of $X)$, we have

$$
\sum_{j=1}^{k-1} d_{j}(a, b)=|N(a) \cap N(b)| \geq 2 \delta(G)-(n-2) \geq b(H) .
$$

Suppose that $d_{j}(a, b) \leq 1$ for each $1 \leq j \leq k-1$. Then we will find an edge cut in $H$ with more than $\sum_{j=1}^{k-1} d_{j}(a, b)$ edges, a contradiction to (5.3). Let $E^{\prime}$ be the set of edges $e_{j}$ in $H$ such that an internal vertex of $P_{j}$ contains a vertex of $N(a) \cap N(b)$. Let $V^{\prime}$ be the set of vertices $u^{0}$ in $H$ such that the vertex $f\left(u^{0}\right)$ (i.e., the branching vertex in $G$ corresponding to $\left.u^{0}\right)$ is in $N(a) \cap N(b)$. Recall that $x, y \notin N(a) \cap N(b)$. By our assumption, no vertex in $V^{\prime}$ is incident to an edge in $E^{\prime}$, and for each $e_{j} \in E^{\prime}$, the path $P_{j}$ contains exactly one vertex of $N(a) \cap N(b)$. Thus, it is enough to find in $H$ an edge cut of size greater than $\left|E^{\prime}\right|+\left|V^{\prime}\right|$.

Let $V_{0}$ denote the set of vertices in all components of $H$ containing at least one edge of $E^{\prime} \cup\left\{e_{k}\right\}$ and let $H_{0}$ be the subgraph of $H$ induced by $V_{0}$. Again by Lemma 10 , for each $e_{j} \in E^{\prime}$, either $u_{j} \in A^{\prime \prime}-B^{\prime \prime}$ and $v_{j} \in B^{\prime \prime}-A^{\prime \prime}$ or $v_{j} \in A^{\prime \prime}-B^{\prime \prime}$ 
and $u_{j} \in B^{\prime \prime}-A^{\prime \prime}$. Recall that $x=f\left(u_{k}^{0}\right), y=f\left(v_{k}^{0}\right), x \in A^{\prime \prime}-B^{\prime \prime}$, and $y \in B^{\prime \prime}-A^{\prime \prime}$. It follows that the set $E^{\prime} \cup\left\{e_{k}\right\}$ is contained in an edge-cut in $H$. Let $V_{1}$ and $V_{2}$ be the disjoint subsets of $V\left(H_{0}\right)$ such that

(a) each edge in $E^{\prime} \cup\left\{e_{k}\right\}$ is incident to a vertex in $V_{1}$ and a vertex in $V_{2}$, and

(b) each vertex in $V_{1} \cup V_{2}$ is incident to an edge in $E^{\prime} \cup\left\{e_{k}\right\}$.

By the above, $V^{\prime} \cap\left(V_{1} \cup V_{2}\right)=\emptyset$ and hence $\left|V(H)-\left(V_{1} \cup V_{2}\right)\right| \geq\left|V^{\prime}\right|$. If $V_{1} \cup V_{2} \neq V_{0}$, then there is a vertex $u^{0} \in V_{0}-\left(V_{1} \cup V_{2}\right)$ adjacent to $V_{1} \cup V_{2}$. If $u^{0}$ is adjacent to $V_{1}$, then we add $u^{0}$ to $V_{2}$, otherwise add it to $V_{1}$. In any case the number of edges between the new $V_{1}$ and $V_{2}$ is greater than between the old ones. We continue adding vertices to $V_{1} \cup V_{2}$ so that with each added vertex, the number of edges between $V_{1}$ and $V_{2}$ grows by at least one until we add all vertices of $V_{0}-\left(V_{1} \cup V_{2}\right)$. When we add the last vertex of $H_{0}$, we get a partition $\left(V_{1}, V_{2}\right)$ of $V_{0}$ such that the number of edges between $V_{1}$ and $V_{2}$ is at least

$$
\left|E^{\prime} \cup\left\{e_{k}\right\}\right|+\left|V_{0}-\left(V_{1} \cup V_{2}\right)\right| \geq\left|E^{\prime}\right|+1+\left|V^{\prime} \cap V_{0}\right| .
$$

If $H_{0}=H$, then we get a contradiction to (5.3). If $H_{0} \neq H$, then every component $H_{i}$ of $H-V_{0}$ has an even cycle and by Lemma $4, H_{i}$ has an edge cut with at least $\left|V\left(H_{i}\right)\right|$ edges. This together with the partition $\left(V_{1}, V_{2}\right)$ of $V_{0}$ will give an edge cut of $H$ with at least $\left|E^{\prime}\right|+1+\left|V^{\prime} \cap V_{0}\right|+\left|V(H)-V_{0}\right| \geq\left|E^{\prime}\right|+1+\left|V^{\prime}\right|$ edges, a contradiction to $(5.3)$.

Lemma 13. Let $\mathcal{C}$ be optimal, $1 \leq j \leq k-1$. Then there is at most one $a \in A$, such that there is more than one $b \in B$ with $j=j(a, b)$.

Proof. Let $P_{j}=\left(w_{0}, w_{1}, \ldots, w_{p}\right)$, where $w_{0}=u_{j}$ and $w_{p}=v_{j}$. Assume to the contrary that there are $a_{1}, a_{2} \in A$ and $b_{1}, b_{2}, b_{3}, b_{4} \in B$ such that $j\left(a_{1}, b_{1}\right)=$ $j\left(a_{1}, b_{2}\right)=j\left(a_{2}, b_{3}\right)=j\left(a_{2}, b_{4}\right)=j$, where $a_{1} \neq a_{2}, b_{1} \neq b_{2}, b_{3} \neq b_{4}$. By Lemma 10 , we may assume that $u_{j} \in A^{\prime \prime} \backslash B^{\prime \prime}$ and $v_{j} \in B^{\prime \prime} \backslash A^{\prime \prime}$.

Since $\beta_{j}+\gamma_{j} \leq 1$, there exists $i, 1 \leq i \leq p-1$, such that $w_{i} \in N\left(a_{1}\right) \cap N\left(b_{1}\right)$. Since $b_{3} \neq b_{4}$, we may assume that $b_{3} \neq b_{1}$. By Lemma 11 , no vertex in $V\left(P_{j}\right)-w_{i}$ can belong to $N\left(a_{2}\right) \cap N\left(b_{3}\right)$. However, this contradicts the fact that $d_{j}\left(a_{2}, b_{3}\right)>1$.

By Lemma $7,|A|+|B|>2 k$. We may assume that $|A| \leq|B|$. Thus $|B| \geq k$. If $|A| \geq k$, then since $|B| \geq k$, for each $a \in A$ there is some $j(a)$ and $b_{1}(a)$ and $b_{2}(a)$ such that $j(a)=j\left(a, b_{1}(a)\right)=j\left(a, b_{2}(a)\right)$. Furthermore, since $|A| \geq k$, for some $a_{1}, a_{2} \in A$, the indices $j\left(a_{1}\right)$ and $j\left(a_{2}\right)$ are the same. This contradicts Lemma 13.

Thus we may assume that $|A|<k$. Since $|B| \geq k$, for each $a \in A$ there is some $j(a)$ and $b_{1}(a)$ and $b_{2}(a)$ such that $j(a)=j\left(a, b_{1}(a)\right)=j\left(a, b_{2}(a)\right)$. Let $J=\{j(a) \mid a \in A\}$. By Lemma 13, the indices $j(a)$ are distinct for distinct $a \in A$ and hence $|J|=|A|$.

Lemma 14. Suppose that $j \in J$. Then $x$ is not adjacent to some interior vertex of $P_{j}$.

Proof. Let $P_{j}=\left(w_{0}, w_{1}, \ldots, w_{p}\right)$, where $w_{0}=u_{j}$ and $w_{p}=v_{j}$. By the definition of $J$, there exists $a \in A$ and $b_{1}, b_{2} \in B$ such that $d_{j}\left(a, b_{1}\right), d_{j}\left(a, b_{2}\right)>1$. Since $\beta_{j}+\gamma_{j} \leq 1$, this implies that $p \geq 2$. Assume that $u_{j} \in A^{\prime \prime}-B^{\prime \prime}$ and $v_{j} \in B^{\prime \prime}-A^{\prime \prime}$.

Since $u_{j} \notin B^{\prime \prime}$, we may assume that $u_{j} b_{1} \notin E(G)$. Let $w_{i^{\prime}}, w_{i^{\prime \prime}} \in N(a) \cap$ $N\left(b_{1}\right)$ and $i^{\prime}<i^{\prime \prime}$. By our choice of $w_{i^{\prime}}, 1 \leq i^{\prime} \leq p-1$. If $x w_{i^{\prime}} \in E(G)$, then we get a contradiction to Lemma 11 with $a_{0}=x$, since $w_{i^{\prime \prime}} a \in E(G)$. Thus, $x w_{i^{\prime}} \notin E(G)$.

By Lemma 14, $x$ is not adjacent to at least $|J|$ vertices in $X-W$. It also is not adjacent to itself. Thus, $|N(x) \cap X| \leq|X|-|J|-1 \leq|W|+3 k-2 b(H)+2(k-1)-$ 
$|J|-1 \leq 6 k-2 b(H)-3-|J|$. Since $|J|=|A|=|N(x)-X|$, we get

$$
\frac{n+b(H)}{2}-1 \leq \operatorname{deg}(x) \leq 6 k-2 b(H)-3,
$$

which yields $n \leq 12 k-5 b(H)-4<9.5 k-6.5$, a contradiction. This contradiction proves that an optimal partial $H$-linkage is an $H$-linkage in the case of loopless $H$.

By condition (I) in the definition of a partial $H$-linkage, $|X| \leq|W|-2 b(H)+$ $5 k+3 \leq 5 k+2$.

6. Proof of the general case. As in section 2 , it is enough to consider $H$ that either has no uneven components or is connected and has an odd cycle other than a loop, or has at most two vertices. Let $H$ have $k^{\prime}$ nonloop edges and $k^{\prime \prime}$ loops, in total $k=k^{\prime}+k^{\prime \prime}$ edges. Recall that $n \geq 9.5\left(k_{1}+1\right)$, where $k_{1}=k+c(H)$. Note that $b(H)$ does not depend on $k^{\prime \prime}$, thus $b(H) \geq 0.5 k^{\prime}$.

Let $f: V(H) \rightarrow V(G)$ be an injective mapping and $W=f(V(H))$. Let $E(H)=$ $\left\{e_{j}=u_{j}^{0} v_{j}^{0}: 1 \leq j \leq k\right\}$. We may assume that the first $k^{\prime}$ edges are not loops. Let $u_{j}=f\left(u_{j}^{0}\right)$ and $v_{j}=f\left(v_{j}^{0}\right)$.

Let $H^{\prime}$ be the multigraph obtained from $H$ by deleting all loops and let $k_{1}^{\prime}=$ $k^{\prime}+c\left(H^{\prime}\right)$. Since $H^{\prime}$ is loopless, our theorem is proved for it, and thus $f$ can be extended to an $H^{\prime}$-subdivision in $G$ on at most $5 k_{1}^{\prime}+2$ vertices. If $H^{\prime}$ has an acyclic component, then so does $H$, and hence by the above, $\left|V\left(H^{\prime}\right)\right| \leq 2$. It was observed in section 3 that in this case $G$ has a subdivision of $H^{\prime}$ on at most 3 vertices. Thus, in either case, $f$ can be extended to an $H^{\prime}$-subdivision in $G$ on at most $5 k^{\prime}+2$ vertices. Among such $H^{\prime}$-subdivisions choose one, say, $F_{1}$, with the fewest vertices and let $X_{1}=V\left(F_{1}\right)$. We will extend $F_{1}$ to a partial $H$-subdivision $F$ such that:

$\left(\mathrm{I}^{\prime}\right)$ as many loops as possible are mapped to internally disjoint cycles of length at most 4 , and

(II') among partial $H$-subdivisions satisfying $\left(\mathrm{I}^{\prime}\right)$, the set $X=V(F)$ has the smallest size.

We claim that such a partial $H$-subdivision is actually an $H$-subdivision. Suppose not, then we may assume that $F$ represents the images $g\left(e_{j}\right)$ for $1 \leq j \leq q$, where $k^{\prime} \leq q \leq k-1$.

First we observe that by the minimality of $F_{1}$ and $F$, every vertex outside $X$ has at most 3 neighbors in $g\left(e_{j}\right)$ for each $1 \leq j \leq q$.

Let $e_{q+1}$ be a loop at vertex $u_{q+1}^{0}$ and $u_{q+1}=f\left(u_{q+1}^{0}\right)$. Consider graph $G^{\prime}=$ $G-\left(X-u_{q+1}\right)$.

If $H$ is not an isolated vertex, then every $x \in W$ is in $X_{1}$ (in fact, $x$ belongs to $g\left(e_{j}\right)$ for some $\left.1 \leq j \leq k^{\prime}\right)$, therefore, $u_{q+1}$ has at most $3\left(q-k^{\prime}\right)$ neighbors in $X-X_{1}$ by ( $\left.\mathrm{I}^{\prime}\right)$. If $H$ is an isolated vertex, then $k^{\prime}=0, V(H)=\left\{u_{q+1}\right\}$, and $u_{q+1}$ has at most $2 q$ neighbors in $X$. It follows that

$$
\begin{aligned}
\operatorname{deg}_{G^{\prime}}\left(u_{q+1}\right) & \geq \operatorname{deg}_{G}\left(u_{q+1}\right)-5 k^{\prime}-2-3\left(q-k^{\prime}\right) \geq \frac{n+k^{\prime} / 2}{2}-1-5 k^{\prime}-2-3\left(q-k^{\prime}\right) \\
& \geq \frac{n}{2}-4.75 q-3 \geq \frac{9.5(k+1)}{2}-4.75(k-1)-3 \geq 6.5 .
\end{aligned}
$$

Let $S=N_{G^{\prime}}\left(u_{q+1}\right)$. If some vertices of $S$ are adjacent or have a common neighbor in $G^{\prime}$ other than $u_{q+1}$, then we extend our partial $H$-linkage. If this is not the case, 
then all neighbors in $G^{\prime}$ of vertices in $S$, apart from $u_{q+1}$, are distinct. Thus,

$$
\sum_{s \in S}\left(\operatorname{deg}_{G^{\prime}}(s)-1\right)+|S|+1 \leq n-(|X|-1)
$$

Since $S \cap X=\emptyset$, by the above, $\operatorname{deg}_{G^{\prime}}(s) \geq \operatorname{deg}_{G}(s)-\min \{|X|, 3 q\}$ for every $s \in S$. Thus, (6.1) yields $|S|(\delta(G)-\min \{|X|, 3 q\})+1 \leq n-|X|+1$. Since $|S|>6$, we have

$$
6 \frac{n}{2} \leq 6 \min \{|X|, 3 q\}+n-|X| \leq 15 q+n \leq 15(k-1)+n .
$$

It follows that $2 n<15 k$, a contradiction.

Acknowledgment. We thank the referees for their very helpful comments.

\section{REFERENCES}

[1] M. Ferrara, R. J. Gould, G. Tansey, and T. Whalen, On H-linked graphs, Graphs \& Combinatorics, 22 (2006), pp. 217-224.

[2] R. J. Gould and T. Whalen, Subdivision extendability, Graphs \& Combinatorics, to appear.

[3] H. A. Jung, Eine Verallgemeinerung des n-fachen Zusammenhangs für Graphen, Math. Ann., 187 (1970), pp. 95-103.

[4] H. Kierstead, G. SÁrközy, And S. Selkow, On k-ordered Hamiltonian graphs, J. Graph Theory, 32 (1999), pp. 17-25.

[5] A. Kostochka and G. Yu, On H-linked graphs, Oberwolfach Report, no. 1, (2004), pp. 42-45.

[6] A. Kostochka And G. Yu, Minimum degree conditions for $H$-linked graphs, Discrete Applied Mathematics, to appear.

[7] A. Kostochka and G. Yu, An extremal problem for $H$-linked graphs, J. Graph Theory, 50 (2005), pp. 321-339.

[8] R. Thomas and P. Wollan, An improved linear edge bound for graph linkages, European J. Combin., 26 (2005), pp. 309-324.

[9] D. B. West, Introduction to Graph Theory, 2nd ed., Prentice Hall, Upper Saddle River, NJ, 2001.

[10] T. Whalen, Degree Conditions and Relations to Distance, Extendability, and Levels of Connectivity in Graphs, Ph.D. thesis, Department of Mathematics and Computer Science, Emory University, Atlanta, GA, 2003. 